\title{
Progress in the Linear Beam Dynamics Study of Ionization Cooling Channel*
}

\author{
Kwang-Je Kim** and Chun-xi Wang \\ University of Chicago, 5801 South Ellis Avenue, Chicago, IL 60637
}

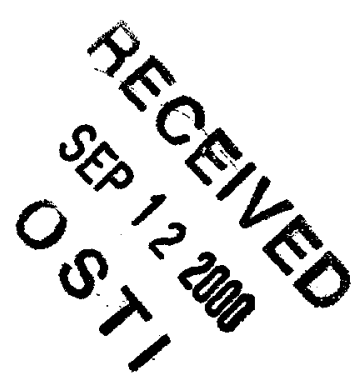

and

Argonne National Laboratory, 9700 South Cass Avenue, Argonne, IL 60439

\begin{abstract}
:
We review recent progresses in the study of linear beam dynamics of the ionization cooling in solenoidal focusing channels. The topics included are: understanding of the solenoidal focusing in the rotating frame; beam parameterization in terms of the envelope functions, emittance, and angular momentum; the coupled equation for emittance and angular momentum in periodic focusing channels; general solution and comparison with simulation; analytic approach for calculating the stability criteria and envelope function in periodic focusing channels.
\end{abstract}

Classification codes: $\quad$ 29.27.-a, 29.27.Bd, 41.75.-i, 41.75.Lx

Keywords: ionization cooling, solenoidal focusing, orbit stability, beam dynamics, envelope and moment equation, muon beam

*Work supported by the U.S. Department of Energy, Office of Basic Energy Sciences, under Contract No. W-31-109-ENG-38.

**Corresponding author: K.-J. Kim, Argonne National Laboratory, Advanced Photon Source, 9700 South Cass Avenue, Building 401, Argonne, Il 60439. Phone: 630-252-4647. Fax: 630252-7369. E-mail: kwangje@aps.anl.gov

The submitted manuscript has been created by the University of Chicago as Operator of Argonne National Laboratory ("Argonne") under Contract No. W-31-109-ENG-38 with the U.S. Department of Energy. The U.S. Government retains for itself. and others acting on its behalf, a paid-up. nonexclusive. irrevocable worldwide license in said article 


\section{DISCLAIMER}

This report was prepared as an account of work sponsored by an agency of the United States Government. Neither the United States Government nor any agency thereof, nor any of their employees, make any warranty, express or implied, or assumes any legal liability or responsibility for the accuracy, completeness, or usefulness of any information, apparatus, product, or process disclosed, or represents that its use would not infringe privately owned rights. Reference herein to any specific commercial product, process, or service by trade name, trademark, manufacturer, or otherwise does not necessarily constitute or imply its endorsement, recommendation, or favoring by the United States Government or any agency thereof. The views and opinions of authors expressed herein do not necessarily state or reflect those of the United States Government or any agency thereof. 


\section{DISCLAIMER}

Portions of this document may be illegible in electronic image products. Images are produced from the best available original document. 


\section{Introduction}

The study of ionization cooling in solenoidal focusing channels proposed for the future muon colliders or neutrino factories [1] presents challenges not encountered in the usual beam dynamics topics. Solenoidal focusing is necessary to capture and transport large emittance beams [2]. Dealing with the nonlinear effects inherent in large-aperture optics is a difficult problem that has so far been addressed only by numerical simulation. The nonlinear problem aside, there are interesting challenges in the linear beam dynamics of the ionization cooling arising from the unique properties of solenoidal focusing: (1) solenoidal focusing is simplest to describe in the rotating "Larmor" frame, while the description of ionization cooling is simpler in the laboratory frame, thus the angular momentum is intimately coupled to the usual beam parameters such as emittance; and (2) thin lens approximation and hence the matrix method is not very useful for solenoidal focusing channels since the solenoidal field continuously occupies the full period. This paper is a review of some recent progress we have made on these problems.

In section 2, we start out with the equation of motion for ionization cooling in the solenoidal focusing channel, showing that the solenoidal focusing becomes the familiar harmonic focusing in the Larmor frame. Working in this frame, we discuss the parameterization of the beam moments in terms of the envelope functions, emittance, and angular momentum. Noting that the interaction with ionization materials is weak, the equations of the envelope function satisfy the same equation as in the beam dynamics of the quadrupole focusing channel. The linear equation describing the coupled evolution of the emittance and angular momentum is solved in the general case and in the case of the periodic channel. These solutions agree well with the results of the simulation calculation. We emphasize the importance of the angular momentum in the evolution and in the limiting value of the emittance. The expression for the 
limiting emittance is the same as the one obtained by Neuffer [3] only when the solenoids of opposite signs are placed symmetrically within each period. Important cases violating this requirement are discussed. The discussion so far has assumed that the beam distribution and the solenoidal field are both cylindrically symmetric. It can be shown that asymmetries in initial beam distribution are damped out to restore the symmetry. This section is mainly based on the works reported in references [4-6].

In section 3 , we discuss the problem of computing the stability criteria and the envelope function for the periodic solenoidal focusing channel. The solenoidal field changes continuously over the length of one period, and the thin lens approximation and hence the usual matrix method is not practical. We discuss a new method based on the results of Hill's equation analysis and derive analytic formulae for stability criteria and envelope function as a power series of Fourier coefficients of the focusing strength. Section 3 is based on the work reported in reference [7].

Beam dynamics study on ionization cooling before the introduction of solenoidal focusing was pioneered by Budker [8], Skrinsky [9], and Neuffer [3], and continued by Fernow and Gallardo [10]. An extensive introduction to ionization cooling in the solenoidal channel can be found in the lecture notes by Palmer and Fernow [2]. Study of linear beam dynamics with solenoidal focusing (using envelope equations) was begun by the Berkely group [11-14]. By identifying the appropriate quantities to characterize the beam dynamics and realizing that the material effects are sufficiently weak, we analytically solved the transverse cooling dynamics for a cylindrical symmetric beam in Ref. [4] and for a general beam in Ref. [6]. Preliminary discussions of the orbit stability problem were given by Fernow [15] and Penn [13]. In Ref. [7] we presented a new analytical approach and formulas that provide solutions for orbit stability and amplitude function $\beta(\mathrm{s})$. These are by no means complete; a good source of recent 
references is the web site for the MUCOOL notes maintained by the mucollider/neutrino factory collaboration [16].

\section{Analysis of Ionization Cooling}

\subsection{Equation of Motion in the Solenoidal Focusing Channel}

Figure 1 shows the schematics of the solenoidal focusing. Let $s$ be the coordinate along the axial direction, and $\mathrm{x}=(\mathrm{x}, \mathrm{y})$ the 2-D transverse coordinates. We use the following expression for the magnetic field:

$$
\mathbf{B}(\mathrm{s}, \mathbf{x})=\mathrm{B}(\mathrm{s}) \mathbf{e}_{\mathrm{s}}-\frac{1}{2}\left(\frac{\mathrm{dB}}{\mathrm{ds}}\right) \mathbf{x}+\text { nonlinear terms } .
$$

Here $B(s)$ is the solenoidal field on axis, $\mathbf{e}_{s}$ is the unit vector along the s-direction. Equation (1) satisfies the divergence of equation $\nabla \cdot \mathbf{B}=0$ exactly and the curl equation $\nabla \times \mathbf{B}=0$ approximately. The field is linear since the nonlinear terms involve higher-order s-derivatives and are neglected assuming slow s-variation. The equation of motion in the laboratory frame is

$$
\frac{\mathrm{d}}{\mathrm{ds}} \mathrm{p}_{\mathrm{s}} \frac{\mathrm{d} \mathbf{x}}{\mathrm{ds}}=-\mathrm{qB} \mathbf{e}_{\mathrm{s}} \times \frac{\mathrm{d} \mathbf{x}}{\mathrm{ds}}-\frac{\mathrm{q}}{2} \frac{\mathrm{dB}}{\mathrm{ds}} \mathbf{e}_{\mathrm{s}} \times \mathbf{x}-\eta \mathrm{p}_{\mathrm{s}} \frac{\mathrm{d} \mathbf{x}}{\mathrm{ds}}+\mathrm{p}_{\mathrm{s}} \mathbf{u} .
$$

Here $\mathrm{p}_{s}$ is the momentum in the s-direction, and $\mathrm{q}$ is the muon's charge. The third term represents the energy loss in the ionization material, where

$$
\eta=\left.\frac{1}{\mathrm{p}_{\mathrm{s}} \mathrm{v}} \frac{\mathrm{dE}}{\mathrm{ds}}\right|_{\text {loss }} \text {. }
$$

Here $v$ is the muon's speed, and $\left.\frac{\mathrm{dE}}{\mathrm{ds}}\right|_{\text {loss }}$ is a positive quantity representing the energy loss per unit length. The last term in Eq. (2) represents the stochastic part of the force due to the multiple scattering in the material. 
It is well known that the precession term $\mathbf{e}_{\mathrm{s}} \times \mathrm{d} \mathbf{x} / \mathrm{ds}$ in Eq. (2) disappears and the motion becomes harmonic in a certain rotating frame [17]: Consider a coordinate system rotating clockwise around the s-axis at an arbitrary rotation rate $\kappa(\mathrm{s})$. The coordinate vector $\mathbf{x}$ in the laboratory frame is related to its rotating frame representation $\mathbf{x}_{\mathrm{R}}$ via

$$
x=R_{R}, \quad R=\left(\begin{array}{cc}
\cos \phi & \sin \phi \\
-\sin \phi & \cos \phi
\end{array}\right),
$$

where $\phi(s)=\int_{0}^{s} \kappa(\bar{s}) d \bar{s}$. Choosing

$$
\kappa(s)=\frac{q B(s)}{2 p_{s}},
$$

the equation of motion in this so-called Larmor frame becomes

$$
\frac{d}{d s} p_{s} \frac{d \mathbf{x}_{R}}{d s}=-p_{s} \kappa^{2} \mathbf{x}_{R}-p_{s} \eta\left(\frac{d \mathbf{x}_{R}}{d s}-\kappa e_{s} \times \mathbf{x}_{R}\right)+p_{s} \mathbf{u}_{R} .
$$

Here, $\mathbf{u}_{\mathrm{R}}=\mathrm{R}^{-1} \mathbf{u}$. Note that the term containing $\mathrm{dB} / \mathrm{ds}$ in Eq. (2) has also disappeared. The part of the equation of motion independent of the material interaction becomes purely harmonic in this frame.

In the following we consider only the case $p_{s}$ is constant. Eq. (5) can then be written as two first-order equations:

$$
\begin{gathered}
\frac{d \mathbf{x}_{\mathbf{R}}}{\mathrm{ds}}=\mathbf{x}_{\mathbf{R}}^{\prime}, \\
\frac{\mathrm{d} \mathbf{x}_{\mathbf{R}}^{\prime}}{\mathrm{ds}}=-\kappa^{2} \mathbf{x}_{\mathbf{R}}-\eta\left(\mathbf{x}_{\mathbf{R}}^{\prime}-\kappa \mathbf{e}_{\mathrm{s}} \times \mathbf{x}_{\mathbf{R}}\right)+\mathbf{u}_{\mathbf{R}} .
\end{gathered}
$$




\subsection{Beam Properties}

Let us now consider the second-order moments that are usually the most important quantities characterizing the beam distribution. If the beam is cylindrically symmetric, there are only four independent moments: $\left\langle\mathbf{x}_{R}^{2}\right\rangle,\left\langle\mathbf{x}_{R}^{\prime 2}\right\rangle,\left\langle\mathbf{x}_{R} \cdot \mathbf{x}_{R}^{\prime}\right\rangle$, and $L=\mathbf{e}_{s} \cdot\left\langle\mathbf{x}_{R} \times \mathbf{x}_{R}^{\prime}\right\rangle$.

$\mathrm{L}$ is the (s-component) angular momentum in the rotating frame, which is also the canonical angular momentum in the laboratory frame: $\mathrm{L}=\mathbf{e}_{\mathrm{s}} \cdot\left\langle\mathbf{x} \times \mathbf{x}^{\prime}\right\rangle+\kappa\left\langle\mathbf{x}^{2}\right\rangle$. In the absence of material $\mathrm{L}$ is conserved as the particle moves in and out of the cylindrically symmetric solenoidal field. Qualitative features of the positively charged particle trajectories in the transverse plane for different signs of $L$ are illustrated in Fig. 2 [18]: For $B_{z}>0$, the motion is clockwise and excludes, passes through, and encircles the origin, according to whether $\mathrm{L}>0, \mathrm{~L}=0$, and $\mathrm{L}<0$, respectively. These properties are reversed for $B_{z}<0$.

We now introduce the emittance and envelope function as in the beam dynamics treatment of quadrupole channels. The emittance is defined as

$$
\varepsilon=\sqrt{\left\langle\mathbf{x}_{\mathbf{R}}^{2}\right\rangle\left\langle\mathbf{x}_{\mathbf{R}}^{\prime 2}\right\rangle-\left\langle\mathbf{x}_{\mathbf{R}} \cdot \mathbf{x}_{\mathbf{R}}^{\prime}\right\rangle}
$$

Note that this emittance is twice the emittance in each transverse direction: $\varepsilon=2 \varepsilon_{\mathrm{x}}$. The beam envelope functions $\beta, \alpha$, and $\gamma$ are defined in the usual way:

$$
\left\langle\mathbf{x}^{2}{ }_{\mathrm{R}}\right\rangle=\varepsilon \beta, \quad\left\langle\mathbf{x}_{\mathbf{R}} \cdot \mathbf{x}_{\mathbf{R}}^{\prime}\right\rangle=-\varepsilon \alpha, \quad\left\langle\mathbf{x}^{2}{ }_{\mathrm{R}}\right\rangle=\varepsilon \gamma .
$$

It then follows that

$$
\beta \gamma-\alpha^{2}=1
$$

Since Eq. (6) are two linear equations, the equations for the second-order moments close in themselves. The moment equations can be converted to the equations for the envelope functions and the beam parameters $\varepsilon$ and $\mathrm{L}$ as follows. The first group of equations is 


$$
\begin{gathered}
\frac{\mathrm{d} \beta}{\mathrm{ds}}+2 \alpha=\eta \beta-\frac{\eta \kappa \mathrm{L}+\chi}{\varepsilon} \beta^{2}, \\
\frac{\mathrm{d} \alpha}{\mathrm{ds}}+\frac{1+\alpha^{2}}{\beta}-\kappa^{2} \beta=-\frac{\eta \kappa \mathrm{L}+\chi}{\varepsilon} \beta \alpha .
\end{gathered}
$$

Here

$$
\chi=\left(\frac{13.6 \mathrm{MeV}}{\mathrm{p}_{\mathrm{s}} \mathrm{v}}\right)^{2} \frac{1}{\mathrm{~L}_{\mathrm{Rad}}}
$$

represents the effect of the stochastic kicks and $\mathrm{L}_{\mathrm{Rad}}$ is the radiation length of the material.

The second group is

$$
\frac{d}{d s}\left(\begin{array}{l}
\varepsilon \\
L
\end{array}\right)=-\left(\begin{array}{cc}
\eta & -\eta \kappa \beta \\
-\eta \kappa \beta & \eta
\end{array}\right)\left(\begin{array}{l}
\varepsilon \\
L
\end{array}\right)+\left(\begin{array}{c}
\beta \chi \\
0
\end{array}\right)
$$

We make an important observation that the right-hand side of Eq. (12) consists of terms representing the interaction of the beam with materials, which is normally weak, and therefore can be set to zero. Equation (10) then becomes the nonlinear differential equation for the beta function, familiar in the beam dynamics of quadrupole channel $[19,17]$ :

$$
\frac{1}{2} \beta^{\prime \prime}+\kappa^{2} \beta-\frac{1}{\beta}\left(1+\frac{\beta^{\prime 2}}{4}\right)=0,
$$

and the relation $\alpha=-\beta^{\prime} / 2$. The envelope function is therefore determined by solving Eq. (13) with the periodic boundary condition, as in the case of the quadrupole focusing case.

\subsection{General Solution}

Having determined the $\beta(\mathrm{s})$, the beam parameter $\varepsilon$ and $\mathrm{L}$ are determined by solving $\mathrm{Eq}$.

(12). We introduce the following notation:

$$
\psi=\left(\begin{array}{l}
\varepsilon \\
L
\end{array}\right), \quad \Xi=\left(\begin{array}{c}
\beta \chi \\
0
\end{array}\right)
$$


The two-component vectors $\psi$ and $\Xi$ will be referred to respectively as the beam state vector and the excitation vector. The general solution of Eq. (12) is

$$
\Psi(s)=\mathrm{e}^{-\hat{\Gamma}(s)} \Psi(0)+\mathrm{e}^{-\hat{\Gamma}(s)} \int_{0}^{s} \mathrm{~d} \bar{s} \mathrm{e}^{\hat{\Gamma}(\bar{s})} \Xi(\overline{\mathrm{s}}),
$$

where

$$
\begin{aligned}
& \hat{\Gamma}(\mathrm{s}) \equiv\left(\begin{array}{cc}
\zeta_{1} & -\zeta_{2}(\mathrm{~s}) \\
-\zeta_{2}(\mathrm{~s}) & \zeta_{1}(\mathrm{~s})
\end{array}\right), \\
& \zeta_{1}(\mathrm{~s}) \equiv \int_{0}^{s} \mathrm{~d} \overline{\mathrm{s}} \eta(\overline{\mathrm{s}}), \quad \zeta_{2}(\mathrm{~s}) \equiv \int_{0}^{s} \mathrm{~d} \overline{\mathrm{s}} \eta(\overline{\mathrm{s}}) \kappa(\overline{\mathrm{s}}) \beta(\overline{\mathrm{s}}) .
\end{aligned}
$$

In ionization cooling channels, the focusing solenoids and absorbers are usually repeated in a periodic array. Let $\lambda$ be the period length. By iterating Eq. (15), one obtains the beam state vector at $\mathrm{s}=\mathrm{m} \lambda, \psi(\mathrm{m} \lambda)$, in terms of the beam state vector at $\mathrm{s}=0, \psi(0)$, and the excitation vector as follows:

$$
\psi(\mathrm{m} \lambda)=\mathrm{e}^{-\mathrm{m} \Gamma(\lambda)} \psi(0)+\left\{1+\mathrm{e}^{-\Gamma(\lambda)}+\mathrm{e}^{-2 \Gamma(\lambda)}+\cdots+\mathrm{e}^{-(\mathrm{m}-1) \Gamma(\lambda)}\right\} \mathrm{e}^{-\Gamma(\lambda)} \int_{0}^{\lambda} \mathrm{ds} \mathrm{e}^{\Gamma(s)}\left(\begin{array}{c}
\beta \chi \\
0
\end{array}\right) .
$$

The first term in Eq. (17) represents the beam state vector at $s=0$ propagated to $s=m \lambda$ while being damped by the factor $\mathrm{e}^{-\mathrm{m} \Gamma(\lambda)}$. The remaining terms are contributions of the excitation generated from the intervening periods.

When the two eigenvalues of $\Gamma(\lambda)$ are both positive, $\exp (-\mathrm{m} \hat{\Gamma}(\lambda)) \rightarrow 0$ as $\mathrm{m} \rightarrow \infty$. In that case the limiting beam state vector is

$$
\psi(\infty)=\frac{\mathrm{e}^{-\hat{\Gamma}(\lambda)}}{1-\mathrm{e}^{-\hat{\Gamma}(\lambda)}} \int_{0}^{\lambda} \mathrm{ds} \mathrm{e}^{\hat{\hat{\Gamma}(s)}}\left(\begin{array}{c}
\beta \chi \\
0
\end{array}\right) .
$$

To the lowest order in $\hat{\Gamma}$, this becomes

$$
\psi(\infty) \approx \frac{1}{\hat{\Gamma}(\lambda)} \int_{0}^{\lambda} d s\left(\begin{array}{c}
\beta(s) \chi(s) \\
0
\end{array}\right)
$$


Using the properties of $\hat{\Gamma}$, this becomes

$$
\left(\begin{array}{l}
\varepsilon(\infty) \\
L(\infty)
\end{array}\right)=\frac{\int_{0}^{\lambda} \beta(s) \chi(s) \mathrm{ds}}{\zeta_{1}^{2}(\lambda)-\zeta_{2}^{2}(\lambda)}\left(\begin{array}{l}
\zeta_{1}(\lambda) \\
\zeta_{2}(\lambda)
\end{array}\right) .
$$

Note from Eq. (16) that $\zeta_{1}(\lambda)=\int_{0}^{\lambda} \mathrm{ds} \eta(\mathrm{s})$ and $\zeta_{2}(\lambda)=\int_{0}^{\lambda} \mathrm{ds} \eta(\mathrm{s}) \kappa(\mathrm{s}) \beta(\mathrm{s})$. The explicit formula for $\psi(m \lambda)$ in terms of initial and equilibrium emittances can be found in Ref. [4].

\subsection{Comparison with Simulation}

The accuracy of the formulas derived here was tested with simulation results using ICOOL [20] and DPGEANT [21] for two cooling channel configurations for the neutrino factory--the 129-m FOFO channel reported by Lebrun et al. [22] and the latest DFOFO channel proposed by Kim [23]. Table 1 shows the cooling factors (the ratio of the beginning to the final transverse emittances) calculated with various methods. For the 3.4-T FOFO channel, there are two data sets: (i) with a $50-\mu \mathrm{m} \mathrm{Al}$ window and (ii) with a 500- $\mu \mathrm{m} \mathrm{Al}$ window. The agreement between our theory and the simulations is remarkable, indicating that the effects of linear beam dynamics dominate the transverse beam properties.

Figure 3 shows the transverse emittances calculated from ICOOL, DPGEANT, and Eq. (15) for the FOFO channel with $50-\mu \mathrm{m} \mathrm{Al}$ windows. Clearly, our theory predicts the simulation results [22] very well, and therefore provides an independent check of the simulations.

However, the simulation predicts somewhat less cooling performance probably due to the fact that it included nonlinearities and longitudinal dynamics. 


\subsection{The Role of Angular Momentum}

Let us delineate the present analysis to the previous studies of ionization cooling. The first quantitative analysis of the ionization cooling was reported by Neuffer [3], who based his analysis on the following equation

$$
\frac{\mathrm{d} \varepsilon}{\mathrm{ds}}=-\eta \varepsilon+\beta \chi
$$

This equation is to be compared with Eq. (12), which would become Eq. (21) if we could neglect the angular momentum. However, the angular momentum effect is important. Thus, the emittance evolution in Fig. 3 can only be calculated by correctly taking into account the angular momentum effect.

In view of Eq. (20), the limiting emittance is given by

$$
\lim _{m \rightarrow \infty} \varepsilon(m \lambda)=\varepsilon^{\infty} \mathscr{F}
$$

where

$$
\varepsilon^{\infty}=\frac{\int_{0}^{\lambda} \mathrm{ds} \beta(\mathrm{s}) \chi(\mathrm{s})}{\int_{0}^{\lambda} \mathrm{d} n \eta(\mathrm{s})} \text {, and } \mathscr{F}=1 /\left(1-\left[\int_{0}^{\lambda} \mathrm{d} \mathrm{d} \eta \kappa \beta\right]^{2} /\left[\int_{0}^{\lambda} \mathrm{d} \mathrm{s} \eta\right]^{2}\right)
$$

The correction factor $\mathscr{F}$ becomes unity when $\zeta_{2}=\int_{0}^{\lambda} \mathrm{ds} \eta \kappa \beta$ vanishes. The limiting angular momentum vanishes in this case. The limiting emittance becomes $\varepsilon^{\infty}$, which is essentially the same as the one derived by Neuffer [3].

The advantage of making $\zeta_{2}$ vanish with the alternating solenoid arrangement was discussed by R. Palmer in his lecture notes [2]. 


\subsection{Constant Solenoid}

As an example where $\mathscr{F}>1$, consider the case where the solenoidal field is constant. In that case $\kappa \beta=1$ in view of Eq. (13), and $\mathscr{F}$ must diverge! What is really happening in this case is that the matrix $1-\mathrm{e}^{-\hat{\Gamma}(\lambda)}$ cannot be inverted, and therefore Eq. (18), for example, is meaningless. It is not difficult to carry out the correct analysis in this case [5], and one finds

$$
\begin{gathered}
(\varepsilon+\mathrm{L})_{\mathrm{m}}=(\varepsilon+\mathrm{L})_{0}+\mathrm{m} \int_{0}^{\lambda} \mathrm{ds} \beta \chi(\mathrm{s}), \\
(\varepsilon-\mathrm{L})_{\mathrm{m}}=(\varepsilon-\mathrm{L})_{0} \mathrm{e}^{-2 \mathrm{~m} \zeta_{1}(\lambda)}+\frac{1-\mathrm{e}^{-2 \mathrm{~m} \zeta_{1}(\lambda)}}{1-\mathrm{e}^{-2 \zeta_{1}(\lambda)}} \mathrm{e}^{-2 \zeta_{1}(\lambda)} \int_{0}^{\lambda} \mathrm{ds} \mathrm{e}^{2 \zeta_{1}(s)} \beta \chi(\mathrm{s}) .
\end{gathered}
$$

Therefore the emittance and the angular momentum contain linearly divergent term as $\mathrm{m} \rightarrow \infty$.

The behavior of the emittance as a function of the number of the period $\mathrm{m}$ in the constant solenoid is sketched in Fig. 4. A constant solenoid channel is therefore not an ideal for cooling to minimum emittance. However, it could provide efficient cooling if used for a limited number of periods $m<m_{c}$, where $m_{c} \approx \frac{1}{2 \zeta_{1}(\lambda)} \ln \left(\frac{\varepsilon(0)-L(0)-\varepsilon^{\infty} / 2}{\varepsilon^{\infty} / 2}\right)$. In this region, the evolution of $\varepsilon$ and $\mathrm{L}$ is dominated by the first terms on the right-hand side, and

$$
\begin{aligned}
& \varepsilon \rightarrow \frac{1}{2}(\varepsilon(0)+\mathrm{L}(0)), \\
& \mathrm{L} \rightarrow \frac{1}{2}(\varepsilon(0)+\mathrm{L}(0)) .
\end{aligned}
$$

Equation (25) is obtained for the case where the solenoidal field and the angular momentum are in the same direction (for a positively-charged muon). If the direction of the solenoid is reversed, we have instead

$$
\varepsilon \rightarrow \frac{1}{2}(\varepsilon(0)-L(0))
$$




$$
\mathrm{L} \rightarrow-\frac{1}{2}(\varepsilon(0)-\mathrm{L}(0))
$$

\subsection{Single Flip Channel}

Recently Balbekov [24] proposed a "single flip" cooling scheme consisting of two constant solenoidal channels of opposite signs. See Fig. 5 for a schematic of the single flip channel [25]. It is easy to see how the cooling works based on Eqs. (25) and (26). In the first channel the emittance and angular momentum approach the value $\varepsilon_{1}=\mathrm{L}_{1}=(\varepsilon(0)+\mathrm{L}(0)) / 2$. In the second channel we see from Eq. (26) that

$$
\varepsilon \rightarrow \frac{1}{2}\left(\varepsilon_{1}-L_{1}\right) \rightarrow 0 \quad \text { and } \quad L \rightarrow-\varepsilon .
$$

Note that the angular momentum tends to be positive $L_{1}=\varepsilon_{1}$ in the first channel while it is negative in the second channel. Recalling the discussion in Fig. 2, the particle trajectories in the first channel, where the angular momentum is positive and decreasing, are circles of decreasing radius. When the field is reversed in the second channel the circles become large to enclose the origin, as qualitatively shown in Fig. 5.

\subsection{Asymmetric Beams}

The ionization cooling theory can be generalized to the case where the beams are not cylindrically symmetric, the solenoids being still symmetric [6]. In this case the ten secondorder moments are parameterized as follows

$$
\begin{gathered}
\left(\left\langle\mathrm{x}^{2}\right\rangle,\left\langle x x^{\prime}\right\rangle,\left\langle\mathrm{x}^{\prime 2}\right\rangle\right)=\left(\beta_{\mathrm{x}},-\alpha_{\mathrm{x}}, \gamma_{\mathrm{x}}\right) \varepsilon_{\mathrm{x}}, \\
\left(\left\langle\mathrm{y}^{2}\right\rangle,\left\langle\mathrm{yy}^{\prime}\right\rangle,\left\langle\mathrm{y}^{\prime 2}\right\rangle\right)=\left(\beta_{\mathrm{y}},-\alpha_{\mathrm{y}}, \gamma_{\mathrm{y}}\right) \varepsilon_{\mathrm{y}}, \\
\left\langle\mathrm{xy}^{\prime}-\mathrm{yx}\right\rangle=\mathrm{L},
\end{gathered}
$$




$$
\left(\langle x y\rangle, \frac{1}{2}\left\langle x y^{\prime}+x^{\prime} y\right\rangle,\left\langle x^{\prime} y^{\prime}\right\rangle\right)=\left(\beta_{x y},-\alpha_{x y}, \gamma_{x y}\right) \varepsilon_{x y} .
$$

In the above, we have introduced separate emittances and envelope functions for moments in both $\mathrm{x}$ - and $\mathrm{y}$-directions and also for the crossed moments. For the symmetric solenoidal field, the three envelope functions $\beta_{x}, \beta_{y}$, and $\beta_{x y}$ all satisfy the same differential equation, Eq. (13), but are in general different from each other due to the different initial conditions. However, the filamentation due to the unavoidable nonlinearities will cause the phase-space ellipses to match the lattice ellipse. The result is that the envelope functions become the matched ones with the periodic boundary condition, and the emittances are enlarged to $\varepsilon^{*}$, $\varepsilon^{*}$, and $\varepsilon^{*}$ xy. It can then be shown that the evolution for $\varepsilon=\varepsilon_{x}+\varepsilon_{y}$ and $L$ is the same as before and

$$
\begin{gathered}
\varepsilon_{x y}(s)=\varepsilon^{*}{ }_{x y} \mathrm{e}^{-\zeta_{1}(s)}, \\
\varepsilon_{x}(s)-\varepsilon_{y}(s)=\left[\varepsilon^{*}{ }_{x}-\varepsilon^{*}{ }_{y}\right] e^{-\zeta_{1}(s)} .
\end{gathered}
$$

Therefore the asymmetry in the distribution is eventually damped out. However, the cooling process takes longer. See Ref. [6] for details.

\section{An Analytic Method for Beam Dynamics Calculation of Periodic Focusing Channels}

Our study of ionization cooling led us to reexamine the beam dynamics calculation for the periodic focusing channel [7]. In designing a periodic focusing channel, it is important to have an efficient way of computing the stability boundary and the beam envelope function for a given focusing configuration. In quadrupole channels, where the focusing can usually be

approximated by a few lenses, the matrix method has been very efficient in evaluating the performance of a channel $[19,17]$. In solenoidal channels for muon cooling, the thin lens 
approximation and therefore the matrix method are not suitable. Here we discuss a method based on Fourier expansion of the focusing function. The method is a straightforward adaptation of the results on the study of Hill's equation, starting from the famous memoir in 1886 on luna perigee [26] and later extensions [27-29]. Reference [29] contains many useful results.

However, these results do not appear to be familiar to the beam physics community.

\subsection{Hill's Equation}

Consider the transverse equation of motion in a periodic focusing channel:

$$
\frac{d^{2} x}{d s^{2}}+K(s) x=0 .
$$

The focusing strength is given by $\mathrm{K}(\mathrm{s})=\left(\mathrm{qB}(\mathrm{s}) / 2 \mathrm{p}_{\mathrm{s}}\right)^{2}$ for a solenoidal channel and $\mathrm{K}(\mathrm{s})=$ $\mathrm{qB}_{1}(\mathrm{~s}) / \mathrm{p}_{\mathrm{s}}$ for a quadrupole channel, where $\mathrm{K}_{1}(\mathrm{~s})$ is the field gradient. It is convenient to cast the equation in the dimensionless form

$$
\frac{d^{2} x}{d \zeta^{2}}+\theta(\zeta) x(\zeta)=0
$$

Here $\zeta=\pi \mathrm{s} / \mathrm{L}$ is the rescaled distance variable, $\mathrm{L}$ is the period length of the channel, and

$$
\theta(\zeta)=\left(\frac{L}{\pi}\right)^{2} K\left(\frac{L}{\pi} \zeta\right)
$$

is the dimensionless focusing strength. Introducing the Fourier expansion,

$$
\theta(\zeta)=\sum_{n=-\infty}^{\infty} \theta_{n} e^{i 2 n \zeta}=\theta_{0}+\tilde{\theta}
$$

Here $\theta_{0}$ is the constant part

$$
\theta_{0}=\left(\frac{\mathrm{qL}}{2 \pi \mathrm{p}_{\mathrm{s}}}\right)^{2}\left\langle\mathrm{~B}_{\mathrm{s}}{ }^{2}\right\rangle,
$$

and $\tilde{\theta}$ is the nonconstant part of $\theta(\zeta)$ : 


$$
\tilde{\theta}(\zeta)=\sum_{n=-\infty}^{\infty} \tilde{\theta}_{n} e^{i 2 n \zeta}
$$

where

$$
\tilde{\theta}_{\mathrm{n}}=\theta_{\mathrm{n}} \text { for } \mathrm{n} \neq 0 \text {, and } \tilde{\theta}_{0}=0 \text {. }
$$

An equation of the type (32) or (33) is known as Hill's equation.

\subsection{Stability Criteria}

When $\tilde{\theta}=0$, the motion is a simple harmonic oscillator with the dimensionless

frequency $\sqrt{\theta_{0}}$. For nonvanishing but small $\tilde{\theta}$, Hill's equation develops stopbands, i.e., regions where the motion is unstable, around $\sqrt{\theta_{0}}=$ integer. The general stability criteria of Hill's equation is determined by $[26,29]$

$$
|\Delta|<2
$$

Here

$$
\Delta=2-4 \sin ^{2}\left(\frac{\pi}{2} \sqrt{\theta_{0}}\right) D
$$

where D is known as Hill's determinant:

$$
\mathrm{D}=\left\|\frac{\tilde{\theta}_{\mathrm{n}-\mathrm{m}}}{\| \theta_{0}-4 \mathrm{n}^{2}}+\delta_{\mathrm{nm}}\right\| .
$$

By expanding Hill's determinant $D$ in powers of $\tilde{\theta}_{n}$, one can derive the expansion

$$
\Delta=2 \cos \left(\sqrt{\theta_{0}} \pi\right)+\frac{\pi \sin \sqrt{\theta_{0}} \pi}{2 \sqrt{\theta_{0}}} \sum_{n=1}^{\infty} \frac{\left|\theta_{n}\right|^{2}}{\theta_{0}-n^{2}}+\cdots .
$$


Third- and higher-order terms in $\tilde{\theta}$ can be found in reference [29]. From Eq. (42), it is apparent that $|\Delta|$ could become larger than 2 , and the motion becomes unstable for $\sqrt{\theta_{0}}=\mathrm{n}$. It can be shown by a more detailed analysis that the stopband is centered at

$$
\sqrt{\theta_{0}} \cong n\left(1+\frac{5}{16} \frac{\theta_{\mathrm{n}}^{2}}{\theta_{0}^{2}}\right)
$$

with width $\Delta \sqrt{\theta_{0}} \cong \mathrm{n} \theta_{\mathrm{n}} / \theta_{0}$, which, in view of Eq. (36), implies momentum width in the stopband

$$
\frac{\Delta \mathrm{p}_{\mathrm{z}}}{\mathrm{p}_{\mathrm{z}}} \approx\left|\frac{\theta_{\mathrm{n}}}{\theta_{0}}\right|
$$

Figure 6 gives the stability parameter calculated exactly $(\Delta)$, by the second-order approximation $\left(\Delta_{+2}\right)$, and by the third-order approximation $\Delta_{+3}$ for the case of the SuperFOFO [13] lattice. Note that the approximate formula reproduces the qualitative feature of the stability diagram reasonably well in these approximations.

Recently Balbekov [30] proposed a lattice in which the stopbands are highly suppressed by reducing the Fourier coefficients $\tilde{\theta}_{n}$ for $n \neq 0$. Figure 7 shows the stability diagram for this case. Notice the strong suppression of the stopband region. In this case, the second-order formula (42) is indistinguishable from the exact calculation.

In a FOFO channel the solenoidal magnetic field as a function of $s$ is nearly sinusoidal:

$$
\mathrm{B}(\mathrm{s})=\mathrm{B}_{0} \sin (\pi \mathrm{s} / \mathrm{L}) \text {. }
$$

This corresponds to

$$
\theta(\zeta)=(1-\cos 2 \zeta) \theta_{0}
$$

Therefore the $n= \pm 2$ Fourier components are large: 


$$
\theta_{ \pm 2}=\theta_{0} / 2
$$

To reduce the harmonics contents in $\theta$, one must therefore add harmonics to $B(s)$. Thus with the solenoidal field

$$
\mathrm{B}(\mathrm{s})=\mathrm{B}_{0}\left(\sin \frac{\pi \mathrm{s}}{\mathrm{L}}+\frac{1}{2} \sin \left(\frac{3 \pi \mathrm{s}}{\mathrm{L}}\right)+\ldots\right)
$$

the $\mathrm{n}= \pm 2$ harmonics in $\theta(\zeta)$ is removed. Figure 8 shows the magnetic field profile for $B(s)$ worked out by Balbekov, clearly exhibiting the higher harmonic contents.

\subsection{Envelope Function}

The significance of the stability parameter and Hill's determinant can be clarified by considering the one-period transfer matrix [19]:

$$
\left[\begin{array}{c}
x(s+L) \\
x^{\prime}(x+L)
\end{array}\right]=M(s)\left[\begin{array}{l}
x(s) \\
x^{\prime}(s)
\end{array}\right] \text {. }
$$

The stability parameter $\Delta$ introduced in Eq. (40) is in fact $(\mathrm{Tr}=$ trace $)$

$$
\operatorname{Tr} M(s)=\Delta,
$$

which is a quantity independent of s. The one-period matrix can be calculated in powers of $\tilde{\theta}$ by recursively solving the equation of motion. The expansion, Eq. (42), can be verified in this way. If the stability condition is satisfied, then one can write

$$
\Delta=2 \cos \mu,
$$

where $\mu$ is the phase advance of the transverse motion in one period.

The matrix element $M_{12}$ can also be calculated recursively, giving rise to an explicit expression of the envelope function $\beta(\mathrm{s})$ :

$$
\beta(s)=\frac{\mathrm{L} \sin \left(\sqrt{\theta_{0}} \pi\right)}{\pi \sqrt{\theta_{0}} \sin \mu}\left\{1+\sum_{\mathrm{n}=1}^{\infty} \frac{\operatorname{Re}\left[\theta_{\mathrm{n}} \mathrm{e}^{\mathrm{i} 2 \mathrm{~ns}}\right]}{\mathrm{n}^{2}-\theta_{0}}+\cdots\right\} .
$$


The expression for the second-order term has also been derived [7].

Figure 9 gives a comparison of the envelope function up to the second order with exact results in the case of the SuperFOFO channel. In addition to giving a simple formula for numerical calculation, the approximate but explicit expression for the beta function is useful in getting insights on optimizing the design of a focusing channel. Thus Eq. (51) suggests the following possibilities for minimizing the value of $\beta(s)$ : Minimize $L$; maximize the dimensionless frequency $\sqrt{\theta_{0}}$; maximize the phase advance term $\sin \mu$; choose large $\theta_{\mathrm{n}}$ so that cancellation takes place among the terms within curly brackets in Eq. (51). Of course, these strategies need to be implemented with care since they are often in contradiction with the stability requirements. 


\section{References}

[1] C. Ankenbradt et al., Phys. Rev. ST Accel. Beams 2, 081001 (1999).

[2] R.B. Palmer and R. Fernow, "Beam Physics for Muon Colliders," lectures given at the Accelerator School, Vanderbilt University (1999).

[3] D. Neuffer, Part. Accel. 14, 75 (1983).

[4] K.-J. Kim and C.X. Wang, Phys. Rev. Lett. 85, 760 (2000).

[5] K.-J. Kim and C.X. Wang, "Simple formulae for transverse cooling in a single-flip solenoidal channel," MUCOOL NOTE 113 (2000).

[6] C.X. Wang and K.-J. Kim "Transverse ionization cooling dynamics of an unmatched beam in solenoidal channels," to be published.

[7] C.X. Wang and K.-J. Kim, "New analytical approach for periodic focusing channels," to be published.

[8] G.I. Budker and A.N. Skrinsky, Usy. Piz. Nauk 124, No. 4 (1978).

[9] A.N. Skrinsky, Proc. of International Seminar on Prospects of High-Energy Physics, March, 1971.

[10] R.C. Fernow and J.C. Gallardo, Phys. Rev. E52, 1039 (1995).

[11] C.M. Celata and A.M. Sessler, Proceedings of the 1998 European Particle Accelerator Conference, 1058 (1998).

[12] B.A. Shadwick and J.S. Wurtele, Proceedings of the 1999 Particle Accelerator Conference, 1716 (1999).

[13] G. Penn, "Beam Envelope Equations in a Solenoidal Field," MUCOOL NOTE 71, (2000).

[14] G. Penn and J.S. Wurtele, Phys. Rev. Lett. 85, 764 (2000). 
[15] R.C. Fernow, "Stability of alternating solenoidal lattice solutions," MUCOOL NOTE 26 (1999); (http://www-mucool.fnal.gov/notes/notes.html).

[16] MUCOOL Notes at http://www-mucool.fnal.gov/notes/notes.html.

[17] See, for example, M. Reiser, "Theory and Design of Charged Particle Beams," John Wiley \& Sons, New York, NY (1994).

[18] K. McDonald, "Comments on Ionization Cooling," MuMu/98-17 at http://www.hep.princeton.edu/mumu.

[19] E.D. Courant and H.S. Synder, Ann. of Phys. 3, 1 (1958).

[20] R.C. Fernow, Proceedings of 1999 Particle Accelerator Conference, 3020 (1999), and ICOOL User's Manual.

[21] P. Lebrun, http://www-pat.fnal.gov/muSim/DPGeant.html.

[22] P. Lebrun, J. Monroe, and G. Penn, "Comparison of the Performance of 3.4 T FoFo and 6.3 T SFoFo Channels Simulated in ICOOL and DPGeant," MUCOOL NOTE 68, (1999).

[23] Eun-San Kim, "SFoFo Cooling Channels for a Neutrino Factory," MUCOOL NOTE 79 (2000).

[24] V. Balbekov, P. Lebrun, J. Monroe, and P. Spentzouris, "The Single Field Flip Cooling Channel for a Neutrino Factory," MUCOOL NOTE 125 (2000).

[25] “A Feasibility Study of a Neutrino Source Based on a Muon Storage Ring," edited by N. Holtkamp and D. Finley, March, 2000.

[26] G.W. Hill, Acta. Math. 8, 1-36 (1886).

[27] E.T. Whittaker and G.N. Watson, A Course of Modern Analysis, $4^{\text {th }}$ Ed., Cambridge University Press, 1952. 
[28] P.M. Morse and H. Feshbach, Methods of Theoretical Physics, p. 560, McGraw-Hill Book Company, Inc. (1953).

[29] W. Magnus and S. Winkler, Hill's Equation, John Wiley \& Sons, Inc. (1996).

[30] V. Balbekov, "New design of alternate solenoid cooling channel for Neutrino Factory," MUCOOL NOTE 98 (2000). 
Table 1. Comparison of transverse emittance (cooling factor) calculations for muon cooling channels

\begin{tabular}{|l|c|c|c|}
\hline & 3.4 T FOFO (1) & 3.4 T FOFO (2) & DFOFO \\
\hline$\varepsilon^{\infty}$ & 0.19 & 0.27 & 0.080 \\
\hline$\varepsilon(\mathrm{m} \lambda)$ & 0.21 & 0.34 & 0.095 \\
\hline ICOOL & 0.22 & 0.34 & 0.10 \\
\hline DPGeant & 0.24 & 0.37 & N/A \\
\hline
\end{tabular}




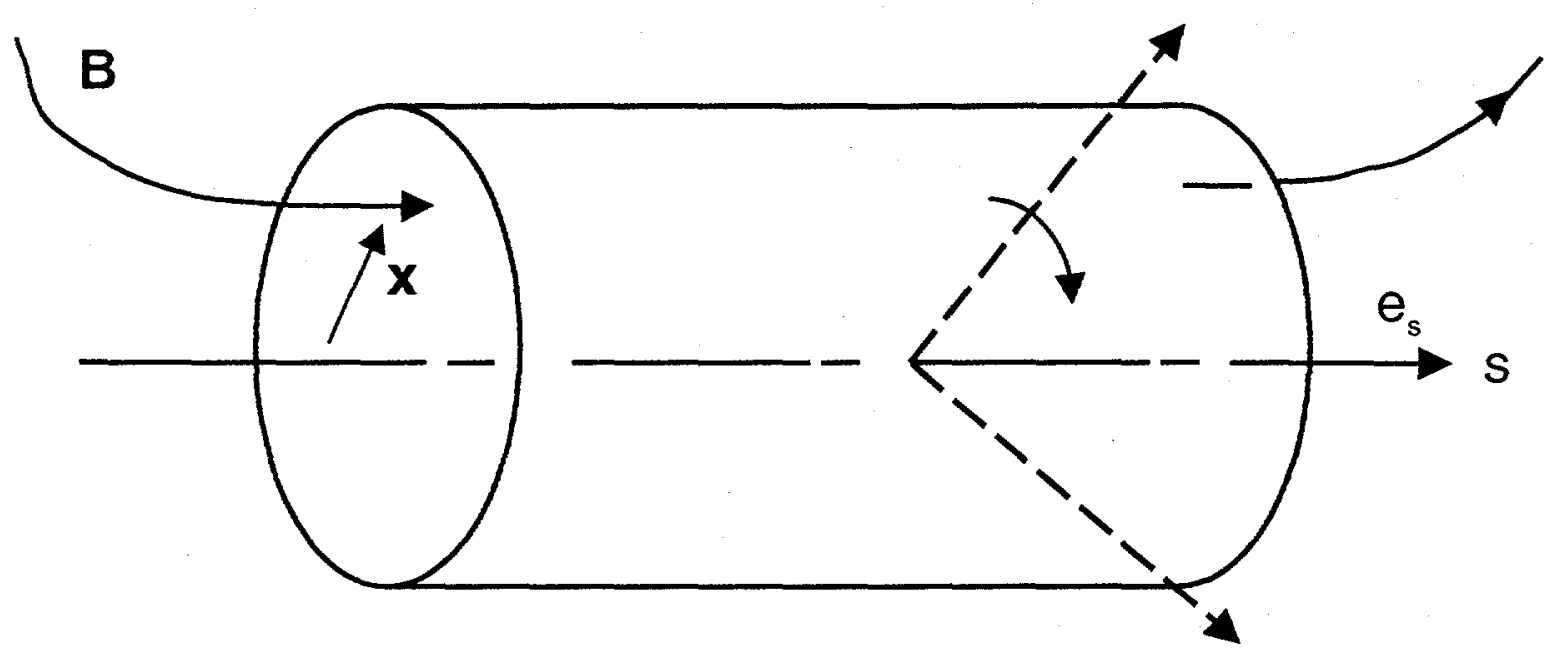

Figure 1. Schematic of a solenoidal focusing channel. 


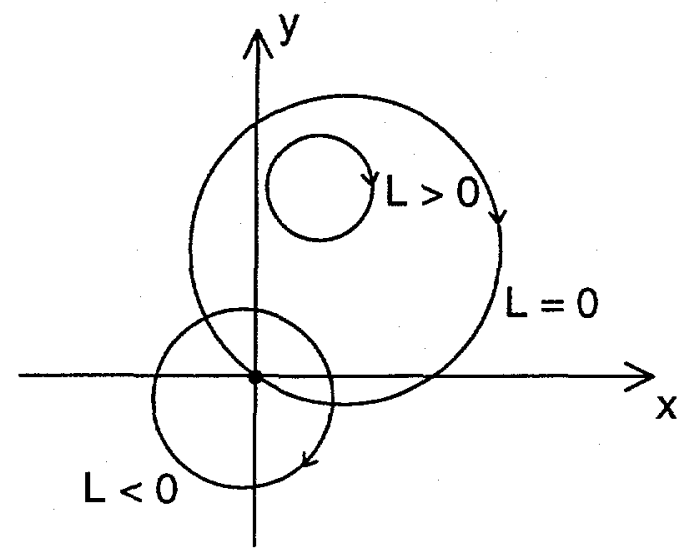

$B_{z}>0$

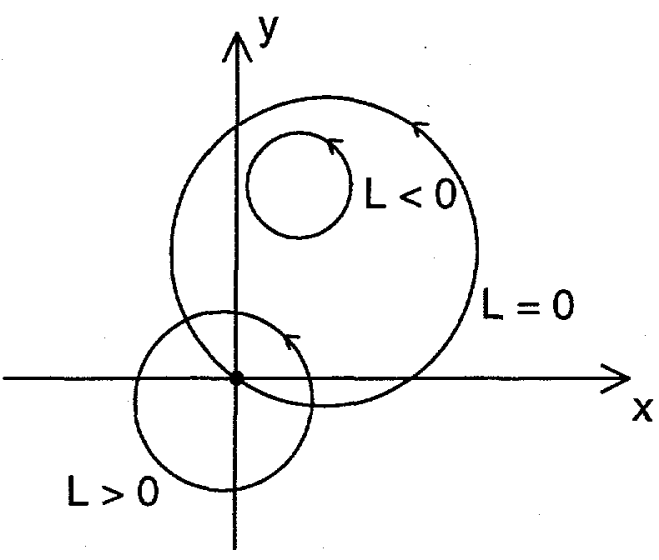

$\mathrm{B}_{2}<0$

Figure 2. Characteristics of positively-charged particle orbits with different angular momenta. 


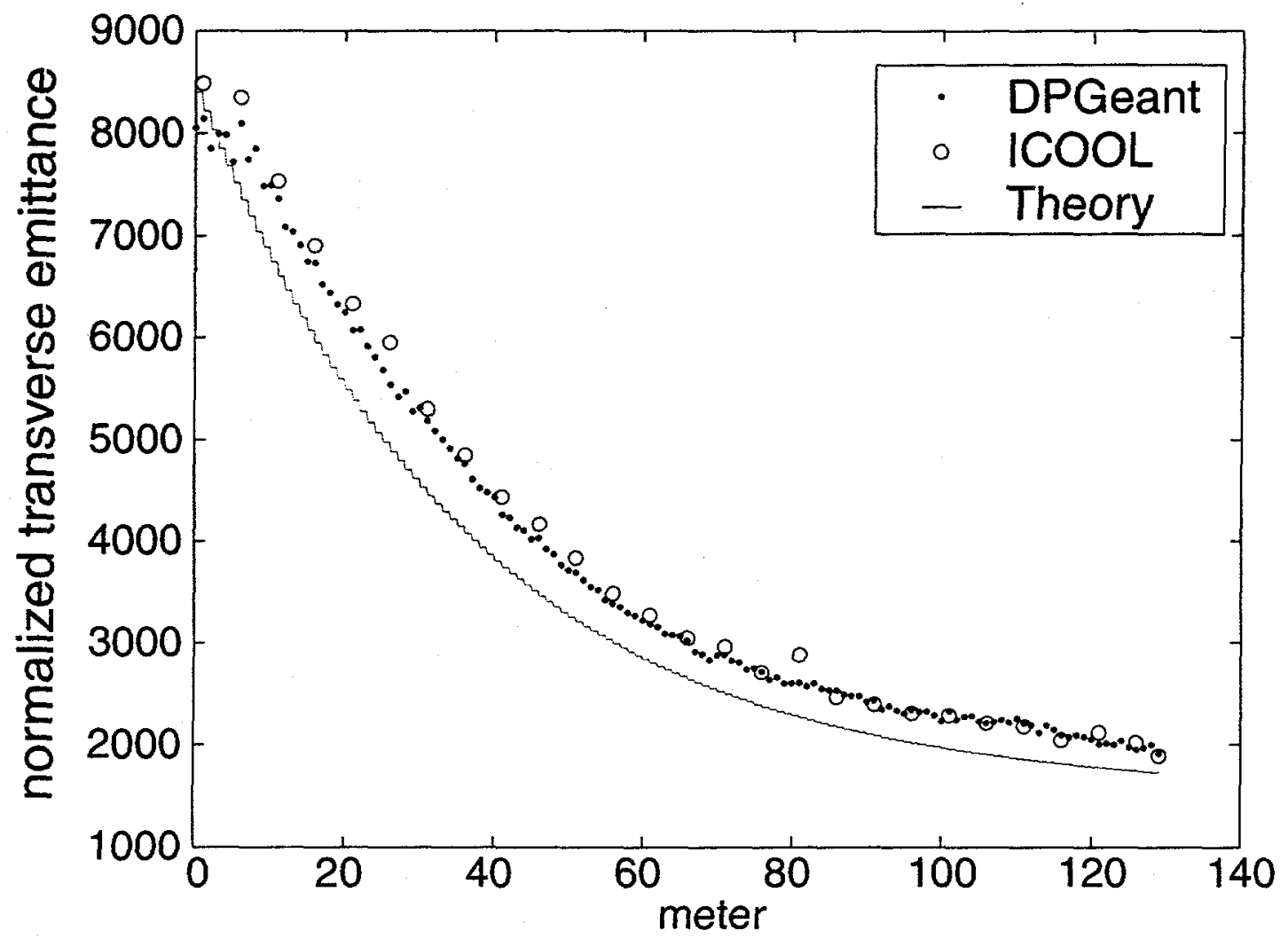

Figure 3. Comparison of cooling performance calculations. 


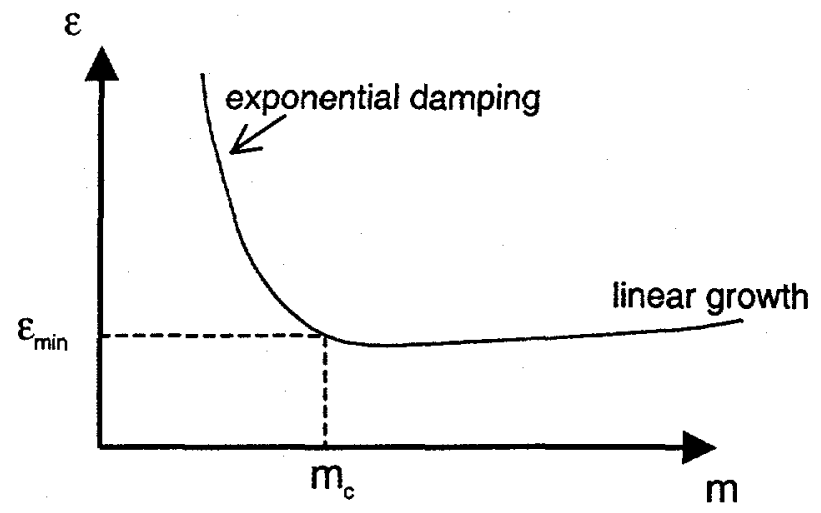

Figure 4. Cooling behavior in a constant solenoid channel. 


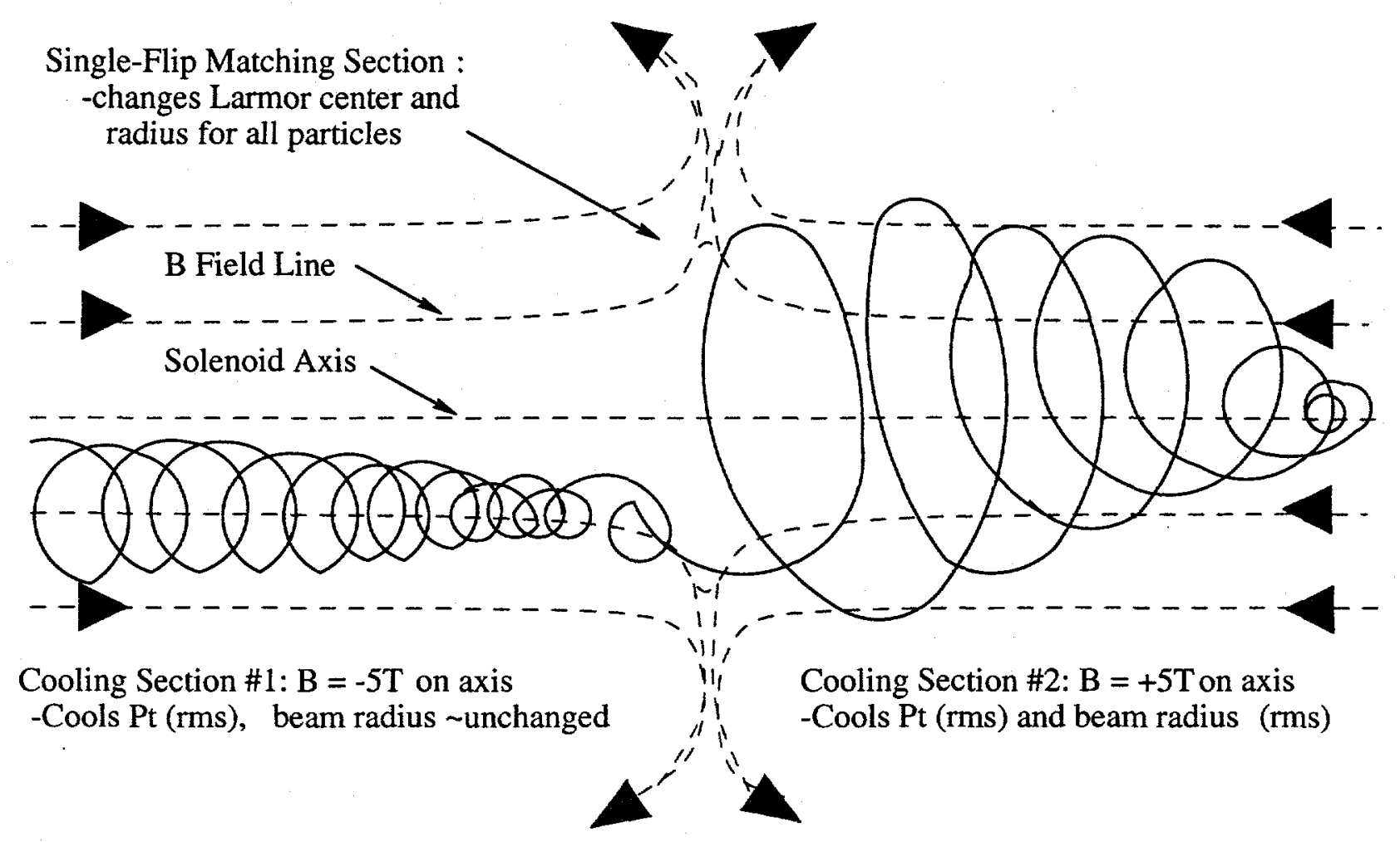

Figure 5: Particle motion cartoon in the single-flip channel.

(Figure courtesy of FNAL Neutrino Factory Design Group) 


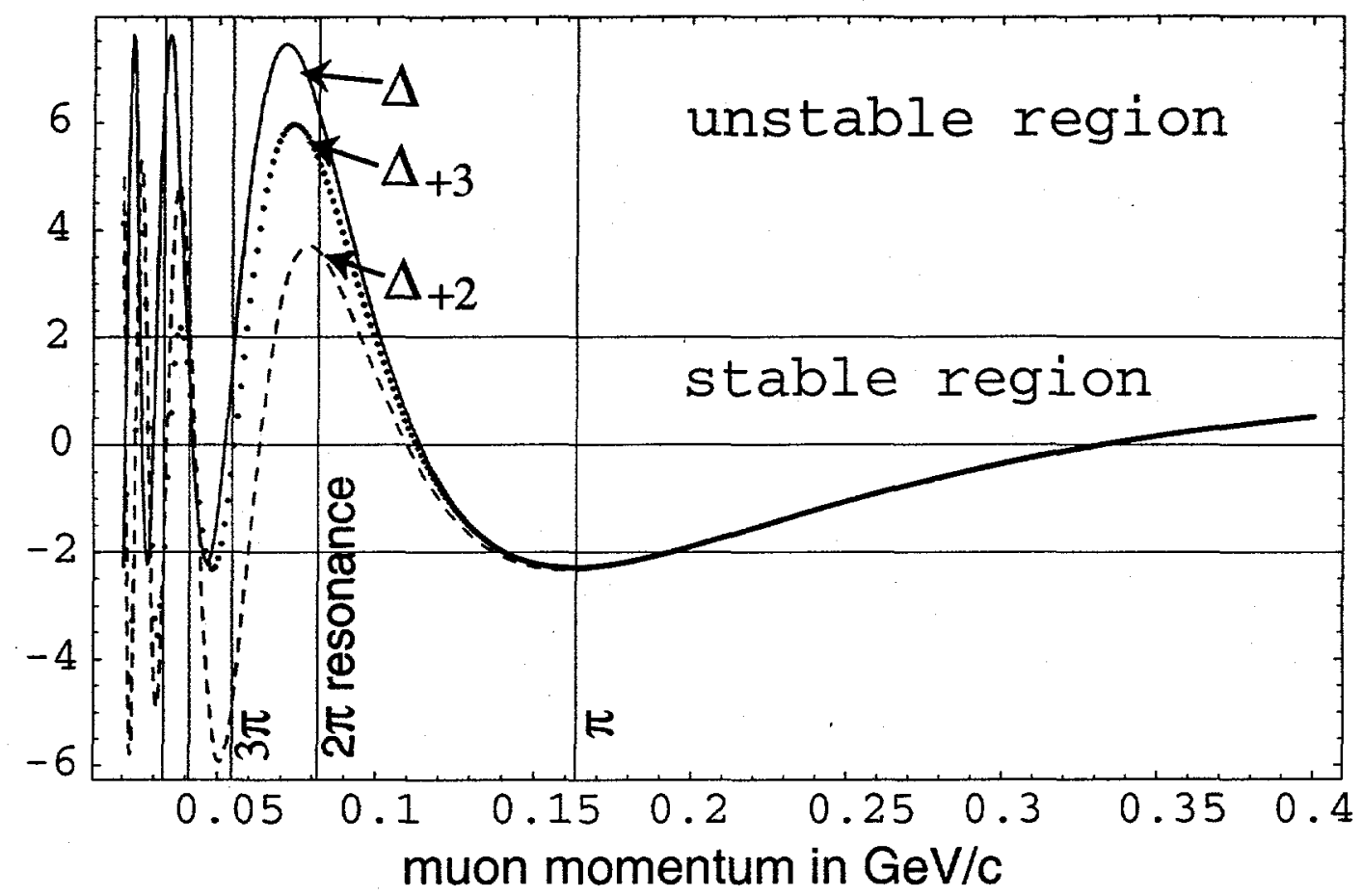

Figure 6. Application to G. Penn's SuperFOFO example. 


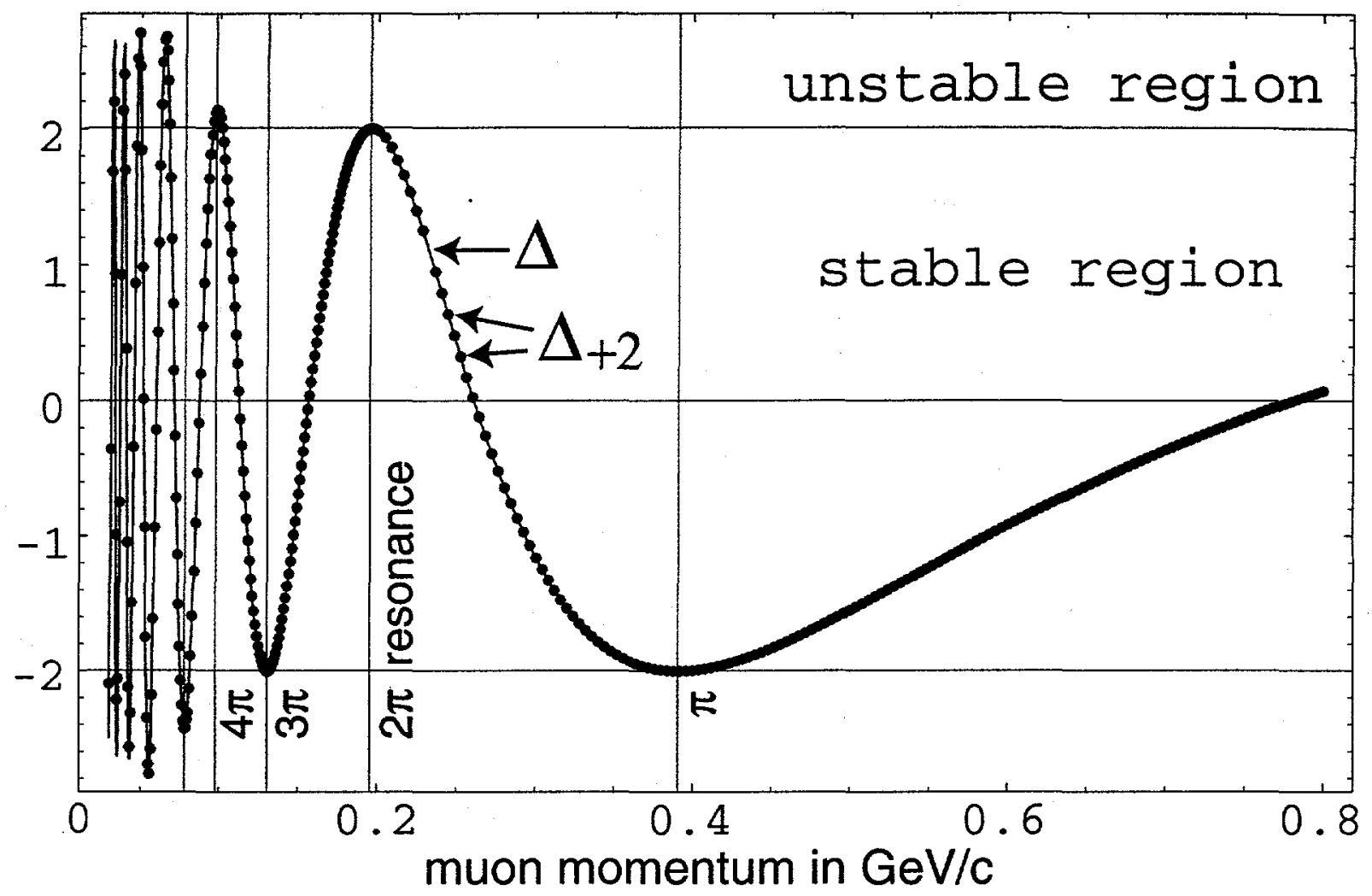

Figure 7. Resonance suppression in V. Balbekov's Case [30]. 

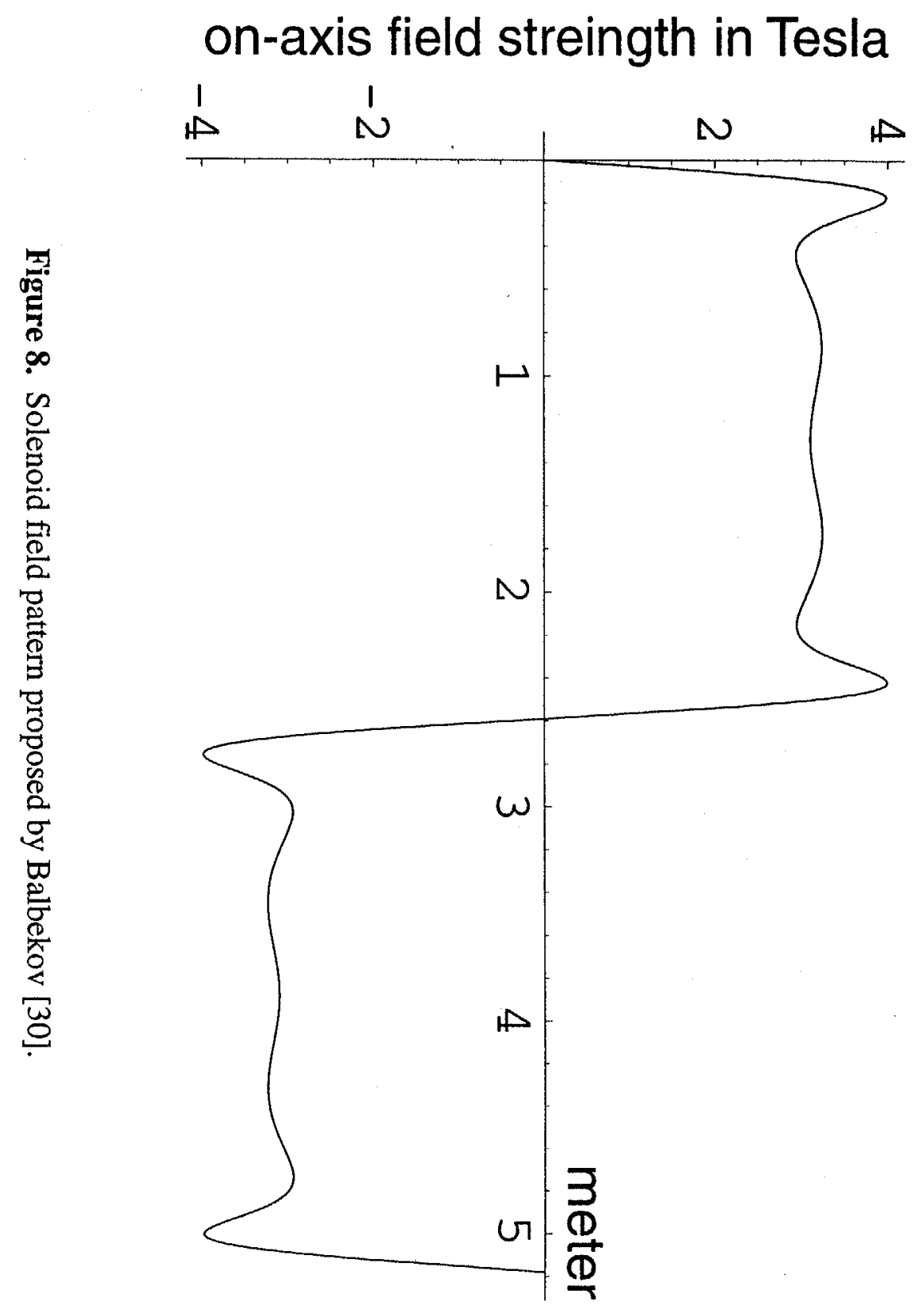


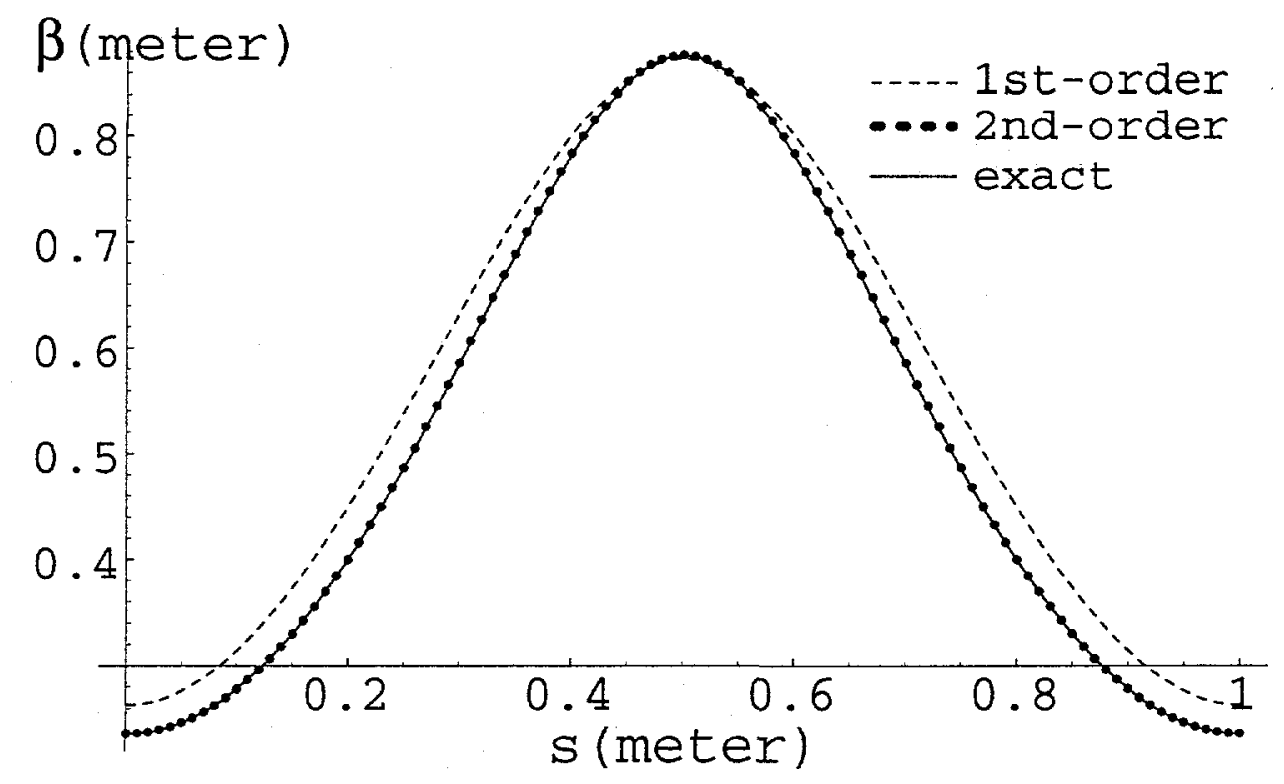

a

b

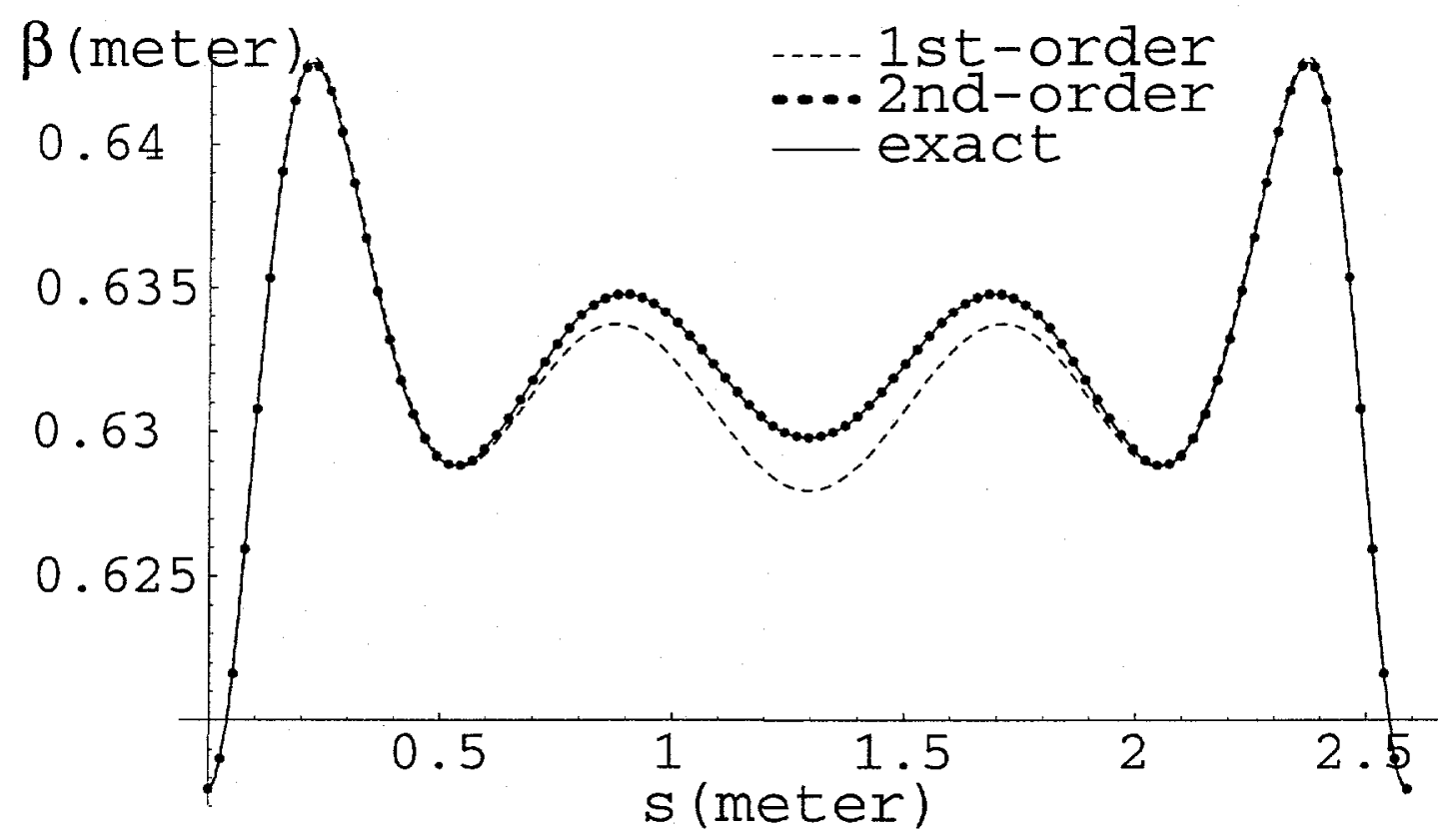

Figure 9. $\beta$-functions calculated using different approximations for

(a) SuperFOFO channel and (b) Balbekov fast-field-flip channel. 